\title{
Efecto protector órgano selectivo de extractos de Psidium guajava L. contra el daño oxidativo en ratas Wistar obesas.
}

\section{Organ-selective protective effect of Psidium guajava L. extracts against oxidative damage in obese Wistar rats}

\author{
Jaaasai Gómez-Otamendi ${ }^{a}$, Nelly S. Cruz-Cansino ${ }^{b}$, Esther Ramírez-Moreno ${ }^{c}$, Zuli Guadalupe \\ Calderón-Rámos ${ }^{d}$, Ernesto Alanís-García ${ }^{e}$, Luis Delgado-Olivares ${ }^{f}$
}

\begin{abstract}
:
Background: During obesity there is an increase of oxidative stress that results in the development of different diseases, so the antioxidants can be used to prevent the oxidative damage. The aim of this study was to analyze the effect of antioxidants in guava extract on the reduction of oxidative damage in different organs of obese rats. Methods: Two extractions were realized to obtain the guava extract (EG), the first one with methanol and the second one with acetone, both were mixed and the activity and content antioxidants were analyzed, the EG was concentrated and used in obese male Wistar rats. Three groups of 8 animals each were formed and fed with a normal diet (Ct), a high-fat diet (AG) and a high-fat diet plus guava fruit extract (AG+EG) during 4 months. Results. The determination of phenolic acid and ascorbic acid of the EG showed a high antioxidant content (3323.0 $\pm 23.3 \mathrm{mg}$ GAE/100g bs and $2508.0 \pm 143.8 \mathrm{mg} \mathrm{AAE} / 100 \mathrm{~g}$ bs, respectively), as well as antioxidant activity determined by ABTS-+ and DPPH- (17500.0 \pm 888.1 $\mu \mathrm{mol} \mathrm{TE} / 100 \mathrm{~g}$ bs and $27804.0 \pm 94.0 \mu \mathrm{mol} \mathrm{TE} / 100 \mathrm{~g}$ bs, respectively). On the other hand, the high-fat diet produced an increase in lipid peroxidation in liver and brain (3.40 \pm 1.05 and 3.99 $\pm 1.49 \mathrm{nmol} \mathrm{MDA} / \mathrm{ml}$, respectively). Fortification of this diet with EG reduced the levels of lipid damage in both liver and heart $(1.94 \pm 0.67$ and $2.16 \pm 1.21 \mathrm{nmol} \mathrm{MDA} / \mathrm{ml}$, respectively). While the brain remained with high levels of lipid peroxidation. Conclusion: The results show that guava extract has a selective protective action against oxidative damage and its use as a potential effect to prevent the development of non-communicable diseases associated with obesity.
\end{abstract}

Keywords:

Antioxidants, guava extract, lipid peroxidation, obesity, oxidative stress.

\section{Resumen:}

Antecedentes: Durante la obesidad hay un aumento del estrés oxidativo que resulta en el desarrollo de diferentes enfermedades, por lo que los antioxidantes se pueden utilizar para prevenir el daño oxidativo. El objetivo de este estudio fue analizar el efecto de los antioxidantes del extracto de guayaba sobre la reducción del daño oxidativo en diferentes órganos de ratas obesas. Métodos: Se realizaron dos extracciones para obtener el extracto de guayaba (EG), la primera con metanol y la segunda con acetona, ambas fueron mezcladas y se analizó la actividad y el contenido antioxidantes, el EG se concentró y se utilizó en ratas macho Wistar obesas. Tres grupos de 8 animales cada uno se formaron y alimentaron con una dieta normal (Ct), una dieta alta en grasas (grupo AG) y una dieta alta en grasas enriquecido con extracto de fruta de guayaba (AG+EG) durante 4 meses. Resultados: La determinación de ácido fenólico y ácido ascórbico del EG, mostró un alto contenido de antioxidantes $(3323.0 \pm 23.3 \mathrm{mg}$ de GAE/100g bs y $2508.0 \pm 143.8 \mathrm{mg}$ de AAE $/ 100 \mathrm{~g}$ bs, respectivamente), al igual que actividad antioxidante determinada por ABTS $^{++}$y DPPH $(17500.0 \pm 888.1 \mu \mathrm{mol} \mathrm{TE} / 100 \mathrm{~g}$ bs y $27804.0 \pm 94.0 \mu \mathrm{mol} \mathrm{TE} / 100 \mathrm{~g}$ bs, respectivamente). Por otro lado, la dieta alta en grasa produjo un incremento en la peroxidación

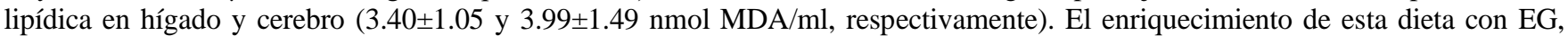

\footnotetext{
a Universidad Autónoma del Estado de Hidalgo, https://orcid.org/0000-0002-9843-9104, Email: go262807@uaeh.edu.mx

b Universidad Autónoma del Estado de Hidalgo, https://orcid.org/0000-0002-6771-3684, Email: ncruz@uaeh.edu.mx

c Universidad Autónoma del Estado de Hidalgo, http://orcid.org/0000-0002-9928-8600, Email: esther_ramirez@uaeh.edu.mx

d Universidad Autónoma del Estado de Hidalgo, https://orcid.org/0000-0002-0263-6067, Email: zramos@ uaeh.edu.mx

e Universidad Autónoma del Estado de Hidalgo, https://orcid.org/0000-0003-1540-4908, Email: ernesto_alanis@uaeh.edu.mx

Autor de Correspondencia, Universidad Autónoma del Estado de Hidalgo, https://orcid.org/0000-0002-3506-8393, Email: 
redujo los niveles del daño a los lípidos tanto en el hígado y como el corazón (1.94 \pm 0.67 y $2.16 \pm 1.21 \mathrm{nmol} \mathrm{MDA} / \mathrm{ml}$, respectivamente). Mientras que el cerebro se mantuvo con altos niveles de peroxidación lipídica. Conclusión: Los resultados muestran que el extracto de guayaba tiene una acción de protección selectiva contra el daño oxidativo y su uso como un efecto potencial para prevenir el desarrollo de enfermedades no transmisibles asociadas con la obesidad.

Palabras Clave:

Antioxidantes, extracto de guayaba, peroxidación lipídica, obesidad, estrés oxidativo

\section{Introducción}

A nivel mundial, cerca de 2.100 millones de personas presentan obesidad, número que aumenta cada año ${ }^{1}$. La obesidad se considera un problema de salud epidémico y un factor de riesgo para el desarrollo de muchas enfermedades no transmisibles, incluyendo 18 comorbilidades entre las que se encuentran la diabetes mellitus, las enfermedades cardiovasculares, los accidentes cerebrovasculares, el cáncer y las enfermedades hepáticas, entre otras ${ }^{2}$. También, hay que considerar que la obesidad se considera uno de los principales componentes del síndrome metabólico ${ }^{3}$, afectando la calidad y la esperanza de vida de las personas 4 . Una posible causa de enfermedades asociadas a la obesidad, es la acumulación de ácidos grasos en el tejido adiposo, los que produce un aumento en la producción de especies reactivas de oxígeno (ERO) mediante la activación de NADPH oxidasa y decremento de la expresión o actividad de enzimas antioxidantes en el tejido adiposo ${ }^{5}$. Por otro lado, la baja ingesta de frutas y verduras ricas en antioxidantes reduce la capacidad de inhibir las moléculas oxidantes que se producen en la célula, llevando a un estado de estrés oxidativo, durante el cual se afecta la estructura y función de proteínas, lípidos y ácidos nucleicos ${ }^{6}$, alterando la función celular, así como de tejidos y órganos lo que conduce al desarrollo de diversa enfermedades ${ }^{7}$.

Por otro lado, la fruta de guayaba o sus extractos han sido considerados como un alimento funcional por su alto nivel de antioxidantes ${ }^{8}$, estos se han utilizado en el tratamiento de diversas patologías como el cáncer, gota, hipertensión y diabetes ${ }^{9-12}$. El objetivo de este estudio fue analizar el efecto de los antioxidantes del extracto de guayaba sobre la reducción del daño oxidativo en diferentes órganos de ratas obesas, con la finalidad de que pueda ser utilizado en la prevención del desarrollo de enfermedades asociadas al estrés oxidativo en condiciones de obesidad.

\section{Material y métodos}

\section{Extracción de muestras y antioxidantes}

La Guayaba (Psidium guajava L.) fue adquirida en un mercado local de Pachuca, Hidalgo, México. Los frutos sin daño externo fueron seleccionados, lavados, cortados en trozos pequeños y liofilizados (Labconco 7753020, USA). Posteriormente, se realizó una molienda y se tamizó para obtener un tamaño de partícula de $500 \mu \mathrm{m}$. La extracción de antioxidantes de guayaba se realizó con el método descrito previamente por Saura-Calixto ${ }^{13}$, con ligeras modificaciones. Se utilizarón $250 \mathrm{mg}$ de guayaba liofilizada, los cuales fueron mezclados con $10 \mathrm{ml}$ de solución de metanol/agua (50:50 v/v), con agitación constante durante $30 \mathrm{~min}$ a $50^{\circ} \mathrm{C}$ (Lab Tech LSI-30106, USA). Al termino la solución fue centrifugada a $1318 \mathrm{Xg}$ durante 10 minutos (Hamilton Van Guard V6500, USA). El sobrenadante fue recuperado, y el pellet fue mezclado con $10 \mathrm{ml}$ de solución de acetona/agua (70:30 v/v) y tratado en las mismas condiciones descritas anteriormente. Después de la centrifugación ambos sobrenadantes se combinaron y ajustaron a $25 \mathrm{ml}$ con una mezcla de metanol/acetona/agua (25:35:40), posteriormente la actividad y el contenido antioxidante fueron determinados. Por otro lado, el disolvente se evaporó en un rotavapor (Büchi, tipo R-200n, Suiza) a 50 ${ }^{\circ} \mathrm{C}$, y el extracto de guayaba (EG) se dispersó en microtubos y almacenamiento a $-36^{\circ} \mathrm{C}$ hasta su uso en el in vivo.

\section{Determinación del contenido antioxidante}

El contenido fenólico total fue analizado por el método descrito previamente por Stinting ${ }^{14}$, usando $100 \mu \mathrm{l}$ de extracto. La absorbancia se registró a $765 \mathrm{~nm}$ utilizando un lector de microplacas (Power Wave XS UV-Biotek, software KC Junior, USA) y los resultados se expresaron como miligramos de ácido gálico (Sigma-Aldrich, USA) equivalentes por $100 \mathrm{~g}$ de base seca (mg EGA/100 g bs). Mientras que, el contenido de ácido ascórbico, se determinó por el método modificado descrito por Dürüst ${ }^{15}$, donde $100 \mu \mathrm{l}$ de extracto se mezclaron con $100 \mu \mathrm{l}$ de buffer de acetato y $800 \mu$ de DCPI (Sigma-Aldrich. USA) y se registraron los valores de absorbancia a $520 \mathrm{~nm}$ 
utilizando un lector de microplacas (Power Wave XS UVBiotek, software KC Junior, USA). Se utilizó como estándar de referencia ácido ascórbico (Sigma-Aldrich, USA), por lo que los resultados se expresaron como miligramos de equivalentes de ácido ascórbico por cada $100 \mathrm{~g}$ de base seca (mg EAA/100 g bs).

\section{Determinación de la actividad antioxidante}

La capacidad antirradical de $\mathrm{ABTS}^{\circ+}$ se determinó de acuerdo a $\mathrm{Re}^{16}$, con ligeras modificaciones. Se tomó una alícuota de $20 \mu \mathrm{l}$ de muestra y se añadieron a $980 \mu \mathrm{l}$ del catión radical $\mathrm{ABTS}^{\circ+}$ diluido con agua desionizada a una absorbancia de $0.70 \pm 0.10$ a $754 \mathrm{~nm}$. La mezcla fue incubaba durante 7 minutos a temperatura ambiente, posteriormente se determinó la absorbancia a $754 \mathrm{~nm}$ en el lector de microplacas (Power Wave XS UV-Biotek, software KC Junior, USA). La capacidad antioxidante se expresó como micromoles de equivalentes de trolox por $100 \mathrm{~g}$ de base seca ( $\mu \mathrm{mol}$ ET/100 g bs).

Para determinar la actividad antirradical se utilizó el radical DPPH (Sigma-Aldrich, USA), como lo describió Morales $^{17}$, con ligeras modificaciones. $100 \mu \mathrm{l}$ de muestra se añadieron a $500 \mu \mathrm{l}$ de solución de $\mathrm{DPPH}^{\bullet}(7.4 \mathrm{mg} / 100$ $\mathrm{ml}$ en metanol), la mezcla se dejó reposar a temperatura ambiente durante $1 \mathrm{~h}$. Se midió la absorbancia a $520 \mathrm{~nm}$ en el lector de microplacas (Power Wave XS UV-Biotek, software KC Junior, USA). La actividad antioxidante se expresó como micromoles de equivalentes de trolox por cada $100 \mathrm{~g}$ de base seca ( $\mu \mathrm{mol}$ ET/100 g bs).

\section{Animales y ensayo in vivo}

Todos los procedimientos con animales se realizaron de acuerdo con la Norma Oficial Mexicana NOM-062-ZOO1999 y fueron aprobados por el Comité Interno para el Cuidado y Uso de Animales de Laboratorio (CICUAL) de la Universidad Autónoma del Estado de Hidalgo (UAEH). Este estudio se registró con número UAEH-DI-ICSA-NTCF-011. Se obtuvieron 24 ratas Wistar machos con 4 semanas de edad (peso 102 a $105 \mathrm{~g}$ ) del Bioterio de la $\mathrm{UAEH}$, las cuales se mantuvieron a $22 \pm 2{ }^{\circ} \mathrm{C}$, con humedad relativa del $45 \%$ y ambiente controlado de ciclos de luz oscura de $12 \mathrm{~h}$ con ad libitum de alimentos y agua. Los animales se dividieron en tres grupos de 8 ratas cada uno y se alimentaron con diferentes dietas: el primer grupo, utilizado como control (Ct), fue alimentado con una dieta normal y el segundo grupo recibió una dieta alta en grasas (AG) y el tercer grupo una dieta alta en grasas enriquecido con extracto de guayaba ( $A G+E G)$.

\section{Dosificación del extracto}

La dosis terapéutica de extracto de guayaba (EG), utilizado en este estudio fue de $520 \mathrm{mg}$ de $\mathrm{GE} / \mathrm{kg}$ de peso corporal/día de acuerdo a lo reportado por Kumar ${ }^{18}$, para lo cual el EG fue suspendido en agua destilada, hasta un volumen máximo de $3 \mathrm{ml}$, y administrado oralmente diariamente al grupo $A G+E G$, a la misma hora durante 4 meses, utilizando una aguja de Gavage, mientras que a los grupos AG y Ct sólo se les administró un volumen igual de agua correspondiente al volumen de EG suministrado.

\section{Preparación del material biológico}

Después de terminar el tratamiento, los animales fueron sacrificados usando una cámara de $\mathrm{CO}_{2}$. El hígado, el corazón y el cerebro fueron retirados y lavados con buffer PBS ( $\mathrm{NaCl} 1.37 \mathrm{M} \mathrm{KH}_{2} \mathrm{PO}_{4} 1.76 \mathrm{M}, \mathrm{KCl} 26.8 \mathrm{mM}$; $\mathrm{Na}_{2} \mathrm{HPO}_{4} 10 \mathrm{mM}, \mathrm{pH} 7.4$ ) y almacenados a $-72{ }^{\circ} \mathrm{C}$ hasta su análisis.

Los diferentes órganos fueron preparados para el análisis de acuerdo a Baños-Gómez ${ }^{19}$, los tejidos fueron cortados con tijeras, y $25 \mathrm{mg}$ de tejido fueron colocados en microtubos de $1.5 \mathrm{ml}$ y homogeneizados (Sonicator Transsonic 460. Elma, Alemania) a $4{ }^{\circ} \mathrm{C}$ con cuatro pulsos de $20 \mathrm{~s}$ cada uno, en búfer de extracción (Tris- $\mathrm{HCl}$ pH 8.8 500 mM, EDTA 10 mM, SDS 5\%, PMSF $10 \mathrm{mM}$ ). Después fueron centrifugados (Mikro 22R, Hettich, Alemania) a $110 \mathrm{Xg}$ durante $10 \mathrm{~min}$ a $4{ }^{\circ} \mathrm{C}$, los sobrenadantes fueron recuperados y almacenados a -80 ${ }^{\circ} \mathrm{C}$ hasta su análisis.

\section{Ensayo de peroxidación lipídica}

Los niveles de malondialdehído (MDA) se determinaron con un kit de ensayo TBARS (Cayman Chemical, Ann Arbor, MI) de acuerdo con las instrucciones del fabricante. La peroxidación lipídica se expresó como nanomoles de MDA por mililitro ( $\mathrm{nmol}$ de MDA/ml).

\section{Análisis estadístico}

Todas las determinaciones fueron analizadas por triplicado y los datos se expresaron como el promedio \pm desviación estándar (SD). Los datos se evaluaron mediante un análisis unidireccional de varianza (ANOVA). Se evaluaron diferencias significativas entre grupos mediante la prueba de Tukey con una importancia de nivel de valores $P<0.05$ utilizando el software estadístico SPSS (versión 15.0; SPSS, 2006, IBM, Westchester, NY, USA). 


\section{Resultados y Discusión}

EI EG presento un contenido apreciable de ácido fenólico y ascórbico (3323.0 $\pm 23.3 \mathrm{mg}$ de GAE/100g bs y 2508.0 $\pm 143.8 \mathrm{mg}$ de $\mathrm{AAE} / 100 \mathrm{~g}$ bs, respectivamente) así como la actividad antioxidante determinada por $\mathrm{ABTS}^{\circ+} \mathrm{y}$ DPPH $^{\circ}(17500.0 \pm 888.1 \mu \mathrm{mol}$ TE/100g bs y $27804.0 \pm$ $94.0 \mu \mathrm{mol} T E / 100 \mathrm{~g}$ bs, respectivamente). Estos resultados fueron más altos en comparación con otros estudios de extractos de guayaba ${ }^{20-22}$, lo cual pudo deberse a los métodos de extracción utilizados, la madurez de la fruta, así como las condiciones de cultivo de las plantas que podrían afectar la presencia de compuestos bioactivos en la planta o en la fruta.

Tabla 1. Incremento de peso corporal por los diferentes tratamientos.

\begin{tabular}{lccc}
\hline \multicolumn{4}{c}{ Peso $(\mathrm{g})$} \\
\hline Peso inicial & $103.5 \pm 7.5$ & $104.7 \pm 5.4$ & AG+EG \\
Peso final & $340.0 \pm 5.2$ & $442.3 \pm 3.2$ & $432.7 \pm 7.1$ \\
NVI (f/i) & 3.29 & 4.22 & 4.24 \\
\%IRC & & 30 & 27 \\
\hline
\end{tabular}

Estos resultados son el promedio de 8 ratas por grupo 土, desviación estándar (DE).

$\overline{\mathbf{N} V I}(\mathbf{f} / \mathbf{i})$, número de veces de incremento del peso final con respecto al peso inicial.

\%IRC, Porcentaje de incremento con respecto al grupo control. Ct, control, AG, dieta alta en grasas; $\mathbf{A G + G E , ~ d i e t a ~ a l t a ~ e n ~}$ grasas enriquecida con extracto de guayaba

Las ratas alimentadas con una dieta $A G$ aumentaron, en promedio 4.22 veces su peso corporal, con respeto a el peso inicial y el $30 \%$ con respecto al grupo de control (Tabla 1). Este tipo de dieta provoco un aumento de la peroxidación lipídica en hígado (3.40 $\pm 1.05 \mathrm{nmol} \mathrm{MDA} / \mathrm{ml})$ y cerebro $(3.99 \pm 1.49 \mathrm{nmol} \mathrm{MDA} / \mathrm{ml})$ con respecto a la dieta control $(2.15 \pm 0.35$ y $3.26 \pm 1.04 \mathrm{nmol} \mathrm{MDA} / \mathrm{ml}$, respectivamente; Figura $1 \mathrm{~A}$ y $\mathrm{B}$ ). Esto último, pudo deberse a que el exceso de grasa aumenta la producción de donadores de electrones en las mitocondrias, facilitando la generación de superóxido y otras $\mathrm{ERO}^{23}$, afectando el metabolismo celular $^{24,5}$ y causando daño hepático, cerebral y cardíaco ${ }^{25-29}$, lo que puede conducir al desarrollo de varias enfermedades. Mientras que, los niveles de oxidación lipídica en el corazón fueron similar al control $(3.66 \pm 1.08$ y $3.36 \pm 0.72 \mathrm{nmol} \mathrm{MDA} / \mathrm{ml}$, respectivamente; Figura $1 \mathrm{C}$ ), lo que probablemente se debido a la que una dieta alta en grasas aumenta la producción de enzimas antioxidantes endógenas en el corazón (catalasa, SOD, GPx), a través de los peroxisomas, como un mecanismo de autoprotección contra el daño oxidativo ${ }^{30}$.

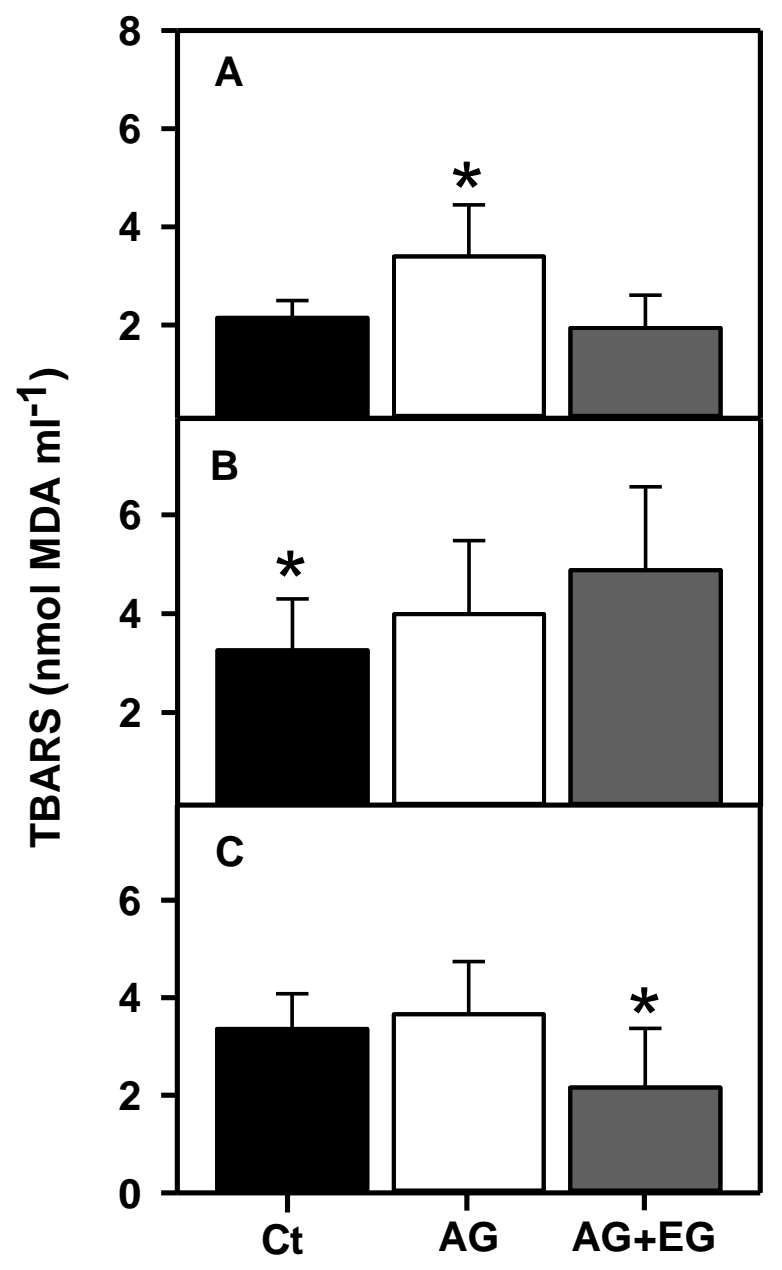

Figura 1. Efecto del extracto de guayaba sobre la peroxidación lipídica en hígado $(A)$, cerebro $(B)$ y corazón (C) de ratas macho alimentadas con una dieta normal $(\mathrm{Ct})$, alta en grasas $(A G)$ alta en grasas adicionada con extracto de guayaba $(A G+E G)$. Valores con $P<0.05$ fueron considerados estadísticamente significativos. Los resultados son el promedio de 8 ratas por grupo.

Por otro lado, la ingesta de la dieta $A G+E G$, no mostró efecto en la reducción del peso corporal (Tabla 1). Sin embargo, si se observó un efecto protector en el hígado y el corazón de las ratas obesas, disminuyendo los niveles de peroxidación lipídica $(1.94 \pm 0.67$ y $2.16 \pm 1.21$ $\mathrm{nmol} \mathrm{MDA} / \mathrm{ml}$, respectivamente), causada por la ingesta de la dieta $A G$ (Figura $1 \mathrm{~A}$ y $\mathrm{C}$ ). Además, el enriquecimiento de la dieta $A G+E G$ permitió que los niveles de MDA en el hígado de rata tuvieran valores similares al grupo de control $(2.15 \pm 0.35 \mathrm{nmol} \mathrm{MDA} / \mathrm{ml})$, incluso en el corazón el EG indujo una reducción de 0.64 veces los niveles normales en comparación con el grupo de control (Figura $1 \mathrm{C}$ ). Esto podría deberse al contenido y tipo de antioxidantes (compuestos fenólicos y ácido ascórbico) presentes en esta fruta ${ }^{31,11}$, previniendo el 
progreso del daño oxidativo ${ }^{32,33}$. Por lo tanto, el extracto de guayaba podría ser utilizado en el tratamiento y prevención de algunas enfermedades del hígado ${ }^{34-36}$ y el corazón ${ }^{37}$ en condiciones de obesidad. Finalmente, el extracto de guayaba no exhibió un efecto protector en el cerebro contra la peroxidación lipídica provocada por los radicales libres producidos por dietas ricas en grasas, lo cual podría deberse a que, aunque cerebro es el órgano más sensible al daño oxidativo ${ }^{38}$ los antioxidantes naturales no pueden cruzar la barrera hematoencefálica ${ }^{39}$.

\section{Conclusiones}

En resumen, el presente estudio mostró que el enriquecimiento con extracto de guayaba en una dieta alta en grasas, puede suprimir o reducir el daño oxidativo en hígado y corazón de ratas obesas, reduciendo los niveles de peroxidación lipídica, debido a los antioxidantes presentes en el extracto de guayaba contrarrestan las ERO producidas por la ingesta de una dieta hipercalórica. Por lo cual, estos resultados apoyan el uso de antioxidantes como agentes terapéuticos en la prevención de enfermedades hepáticas y cardíacas, por lo que, también se pueden utilizar para mejorar la salud de los pacientes obesos y con problemas para bajar de peso. Sin embargo, el hecho de que este efecto no se haya observado en el cerebro, sugiere que el mecanismo de acción de los antioxidantes es diferente en cada órgano; por lo tanto, se necesitan más estudios sobre este tema.

\section{Agradecimientos}

Este trabajo fue apoyado financieramente por el Programa Integral de Fortalecimiento Institucional (PIFI 2012-2013).

\section{Referencias}

[1] Smith KB, Smith MS. Obesity statistics. Prim Care. 2016. 43(1):121135.

[2] Guh DP, Zhang W, Bansback N, Amaris Z, Birmingham CL, Anis AH. The incidence of co-morbidities related to obesity and overweight: A systematic review and meta-analysis. BCM Public Health. 2009. 9(88): $1-20$.

[3] Hanson RL, Imperatore G, Bennett PH, Knowler W C. Components of the "metabolic syndrome" and incidence of type 2 diabetes. Diabetes. 2002. 51(10):3120-3127

[4] Reynolds SL, Saito Y, Crimmins EM. The Impact of obesity on active life expectancy in older American men and women. Gerontologist. 2005. 45(4):438-444.

[5] Matsuda M, Shimomura I. Increased oxidative stress in obesity: implications for metabolic syndrome, diabetes, hypertension, dyslipidemia, atherosclerosis, and cancer. Obes Res Clin Pract. 2013. 7(5):e330-41.

[6] Birben E, Sahiner UM, Sackesen C, Erzurum S, Kalayci O. Oxidative stress and antioxidant defense. World Allergy Organ J. 2012. 5(1):919.

[7] Ogura S, Shimosawa T. Oxidative stress and organ damages. Curr Hypertens Rep. 2014. 16(452):1-5.

[8] Patel P, Ellis K, Sunkara R, Shackelford L, Ogutu Simon, Walker Ll, Herring J, Verghese M. Development of a functional food product using guavas. Food Nut Sci. 2016. 7:927-937.

[9] Bontempo P, Doto A, Miceli M, Mita L, Benedetti R, Nebbioso A, Veglione M, Rigano D, Cioffi M, Sica V, Molinari AM, Altucci L. Psidium guajava L. anti-neoplastic effects: induction of apoptosis and cell differentiation. Cell Prolif. 2012. 45(1):22-31.

[10] Ironi EA, Agboola SO, Oboh G, Boligon AA, Athayde ML, Shode FO. Guava leaves polyphenolics-rich extract inhibits vital enzymes implicated in gout and hypertension in vitro. $\mathrm{J}$ Intercul Ethnopharmacol. 2016. 5(2):122-130.

[11] Musdja MY, Mahendra F, Musir A. Anti-hyperglycemic effect and glucose tolerance of guajava (Psidium guajava L.) leaf ethanol extract in diabetic rats. IOP Conf. Series: Earth and Environmental Science. 2017. 101 (2017) 012006.

[12] Jayachandran M, Vinayagam R, Ambati RR, Xu B, Chung SSM. Guava leaf extract diminishes hyperglycemia and oxidative stress, prevents $\beta$-cell death, inhibits inflammation, and regulates NF-kB signalling pathway in STZ induced diabetic rats. Biomed Res Int. 2018. 18(2018):4601649.

[13] Saura-Calixto F, Serrano J, Goñi I. Intake and bioaccessibility of total polyphenols in a whole diet. Food Chem. 2007. 101(2):492-501.

[14] Stintzing FC, Herbach KM, Mosshammer M R, Carle R. Yi W, Sellapan S, Akoh CC, Bunch R, Felker P. Color, betalain pattern, and antioxidant properties of cactus pear (Opuntia spp.) clones. J Agric Food Chem. 2005. 53(2):442-451.

[15] Dürüst N, Sumengen D, Dürüst Y. Ascorbic acid and element contents of foods of Trabzon (Turkey). J Agric Food Chem. 1997. 45: 20852087.

[16] Re R, Pellegrini N, Proteggente A, Pannala A, Yang M, Rice-Evans C. Antioxidant activity applying an improved ABTS radical cation decolorization assay. Free Radic Biol Med. 1999. 26(9-10):1231-1237.

[17] Morales FJ, Jimenez-Perez S. Free radical scavenging capacity of Maillard reaction products as related to colour and fluorescence. $\mathrm{J}$ Agric Food Chem. 2001. 72:119-125.

[18] kumar A., Kumarchandra R., Rai R., Rao B. S. S., Radiomodulatory Role of Psidium guajava Leaf Extracts against X-ray Induced Genotoxicity, Oxidative stress and Apoptosis in albino Wistar Rat Model. Journal of Applied Pharmaceutical Science. 2016. 6(3):2016.60310.

[19] Baños-Gómez, R., Cruz-Cansino, N.S., Suarez-Diéguez, T., ValadezVega, C., Ramírez-Moreno, E., Alanís-García, E., Ariza-Ortega, J.A., Manríquez-Torres, J.J., Zamora-Romo, E., Delgado-Olivares, L. Undernutrition in the parental and first generation provokes an organspecific response to oxidative stress on neonates of second filial generation of Wistar rats. J Anim Physiol Anim Nutr. 2017. 11(2):267274 
[20] Vasco C, Ruales J, Kamal-Eldin A. Total phenolic compounds and antioxidant capacities of major fruits from Ecuador. Food Chem. 2008. 111:816-823.

[21] Reddy CVK, Sreeramulu D, Raghunath M. Antioxidant activity of fresh and dry fruits commonly consumed in India. Food Res Int. 2019. 43:285-288.

[22] Mesquita J, Patto C, Silveira S, Borges A, Ribeiro A. Evaluation of the protective effect of guava fruits and leaves on oxidative stress. Acta Scientiarum. Biol Sci. 2014. 36(1):35-40.

[23] Rosca MG, Vazquez EJ, Chen Q, Kerner J, Kern TS, Hoppel C L. Oxidation of fatty acids is the source of increased mitochondrial reactive oxygen species production in kidney cortical tubules in early diabetes. Diabetes. 2012. 61(8):2074-2083.

[24] Grattagliano I, Palmieri VO, Portincasa P, Moschetta A, Palasciano G Oxidative stress-induced risk factors associated with the metabolic syndrome: a unifying hypothesis. J Nutr Biochem. 2008. 19(8):491504.

[25] Lozano I, Van der Werf R, Bietiger W, Seyfritz E, Peronet C, Pinget M, Jeandidier N, Maillard E, Marchioni E, et al. High-fructose and high-fat diet-induced disorders in rats: impact on diabetes risk, hepatic and vascular complications. Nutr Metab. 2016. 13:15.

[26] Wang X, Michaelis EK. Selective Neuronal Vulnerability to Oxidative stress in the brain. Front Aging Neurosci. 2010. 2(12):1-13.

[27] Fenkci V, Fenkci S, Yilmazer M, Serteser M. Decreased total antioxidant status and increased oxidative stress in women with polycystic ovary syndrome may contribute to the risk of cardiovascular disease. Fertil Steril. 2003. 80(1):123-127.

[28] Mangge H, Becker K, Dietmar D, Gostner JM. Antioxidants, inflammation and cardiovascular disease. World J Cardiol. 2014. 6(6):462-477.

[29] Pellegrino D. Antioxidants and Cardiovascular Risk Factors. Diseases 2016. $4: 11$.

[30] Chess DJ, Khairallah RJ, O'Shea KM, Xu W, Stanley WC. A high-fat diet increases adiposity but maintains mitochondrial oxidative enzymes without affecting development of heart failure with pressure overload. Am J Physiol Heart Circ Physiol. 2009. 297(5):H1585-93.

[31] Mohamadin AM, Elberry AA, Mariee AD, Morsy GM, Al-Abbasi FA. Lycopene attenuates oxidative stress and heart lysosomal damage in isoproterenol inducedcardiotoxicity in rats: A biochemical study. Pathophysiology. 2012. 19(2):121-30.

[32] Li S, Tan HY, Wang N, Zhang ZJ, Lao L, Wong CW, Feng Y. The role of oxidative stress and antioxidants in liver diseases. Int J Mol Sci. 2015. 16(11):26087-124.

[33] Amiri M. Oxidative stress and free radicals in liver and kidney diseases; an updated short-review. J Nephropathol. 2018. 7(3):127-131.

[34] Yoshitomi H, Guo X, Liu T, Gao M. Guava leaf extracts alleviate fatty liver via expression of adiponectin receptors in SHRP.Z-Leprfa/lzm rats. Nutr Metab. 2012. 9:13.

[35] Peng J, Yue C, Qiu K, Chen J, Aller MA; Ko KS, Yang H. 2013. Protective effects of guava pulp on cholestatic liver injury. ISRN Hepatol. 2013. 17(2013):601071.
[36] Jiao Y, Zhang M, Wang S, Yan C. Consumption of guava may have beneficial effects in type 2 diabetes: A bioactive perspective. Int J Biol Macromol. 2017. 101:543-552.

[37] Yamashiro S, Noguchi K, Matsuzaki T. Cardioprotective effects of extracts from Psidium guajava L. and Limonium wrightii, Okinawan medicinal plants, against ischemia-reperfusion injury in perfused rat hearts. Pharmacology. 2003. 67:128-123.

[38] Uttara B, Singh AV, Zamboni P, Mahajan R. Oxidative stress and neurodegenerative diseases: A review of upstream and downstream antioxidant therapeutic options. Curr Neuropharmacol. 2009. 7(1):6574.

[39] Gilgun-Sherki Y, Melamed E, Offen D. Oxidative stress inducedneurodegenerative diseases: the need for antioxidants that penetrate the blood brain barrier. Neuropharmacology. 2001. 40(8):959-975. 